\title{
LIE-MODEL FOR THOM SPACES OF TANGENT BUNDLES
}

\author{
YVES FÉLIX, JOHN OPREA, AND DANIEL TANRÉ
}

(Communicated by Michael A. Mandell)

\begin{abstract}
We describe the rational homotopy type of Thom spaces and use this information to create a Quillen Lie-model in the case of the tangent bundle of a closed, oriented, simply-connected manifold. Examples are given.
\end{abstract}

In this paper, we describe algebraic models for Thom spaces of bundles, which appear, in particular, as the homotopy cofiber of the canonical injection $F(M, 2) \hookrightarrow$ $M \times M$ of the configuration space of two points in a manifold $M$. Our aim is the determination of the rational homotopy type of the cofiber map $\rho: M \times M \rightarrow$ $\operatorname{Th}(T M)$ in the case of a closed, oriented, simply-connected manifold $M$. This description leads to a conjectural Lie-model of the configuration space of two points in $M$.

Lambrechts and Stanley have already given ([7]) a Sullivan model of $F(M, 2)$, based on the existence of Sullivan models of $M$ satisfying Poincaré duality. The advantages of minimal Lie-models of a manifold are that Poincaré duality is present at the level of the generators and that such a model gives directly a presentation of the rational homotopy Lie algebra of $F(M, 2)$. This existence of Poincaré duality on homology allows an explicit Lie version of the Thom isomorphism and we can construct the model of $M \times M \rightarrow \operatorname{Th}(T M)$ directly from the initial conditions of the problem, the minimal Lie-model of $M$ and the intersection product on homology; see Theorem 3.2. We now briefly describe the contents of the paper.

In 81, we briefly recall the Thom isomorphism, specifying sign conventions and notation. In particular, we express (see Proposition 1.2) the canonical map $\rho_{*}: H_{*}(M \times M) \rightarrow H_{*}(\operatorname{Th}(T M))$ in terms of the intersection product.

In $\$ 2$, we give an explicit description of the rational homotopy type of the Thom space of any vector bundle (see Theorem 2.4) over a closed, oriented, nilpotent manifold in terms of the Euler class of the bundle.

Let $\mathcal{L}_{M \times M}$ and $\mathcal{L}_{\mathrm{Th}(T M)}$ be the Lie-models of $M \times M$ and $\operatorname{Th}(T M)$, respectively. We prove, in 83 , that the projection $\rho: M \times M \rightarrow \operatorname{Th}(T M)$ has a Lie-model which is entirely determined by the map induced in homology, $\rho_{*}$. Finally, in $₫ 4$, we display some concrete examples of this Lie-model.

Received by the editors September 10, 2014 and, in revised form, May 9, 2015.

2010 Mathematics Subject Classification. Primary 55P62; Secondary 55R25.

The first author was partially supported by the MICINN grant MTM2010-18089.

The second author was partially supported by a grant from the Simons Foundation (\#244393).

The third author was partially supported by the MICINN grant MTM2010-18089, the ANR11-BS01-002-01 "HOGT" and the ANR-11-LABX-0007-01 "CEMPI". 
As we are concerned with rational homotopy type, all homologies and cohomologies are taken with coefficients in $\mathbb{Q}$. The degree of an element, $a$, of a graded vector space is denoted by $|a|$.

\section{Poincaré Duality and Thom's isomorphism Revisited}

Let $N$ be a closed, connected, oriented manifold with fundamental class $[N] \in$ $H_{n}(N)$. Poincaré duality is the isomorphism generated by the cap product with $[N]$,

$$
-\cap[N]: H^{n-k}(N) \longrightarrow H_{k}(N) .
$$

We denote by $\mathcal{D}_{N}$ the inverse of $-\cap[N]$, which is thus characterized by

$$
\mathcal{D}_{N}(a) \cap[N]=a, \text { for all } a \in H_{*}(N) .
$$

If we orient the manifold $N \times N$ by the cross product $[N] \times[N] \in H_{2 n}(N \times N)$, we have, for any $a \in H_{|a|}(N), b \in H_{|b|}(N)$,

$$
\begin{aligned}
a \times b & =\left(\mathcal{D}_{N}(a) \cap[N]\right) \times\left(\mathcal{D}_{N}(b) \cap[N]\right) \\
& =(-1)^{n(n-|b|)}\left(\mathcal{D}_{N}(a) \times \mathcal{D}_{N}(b)\right) \cap[N \times N],
\end{aligned}
$$

which implies

$$
\mathcal{D}_{N \times N}(a \times b)=(-1)^{n(n-|b|)} \mathcal{D}_{N}(a) \times \mathcal{D}_{N}(b) .
$$

The intersection product of two homology classes is defined as in [3] by

$$
\begin{aligned}
a \pitchfork b & =\mathcal{D}_{N}^{-1}\left(\mathcal{D}_{N}(a) \smile \mathcal{D}_{N}(b)\right) \\
& =\left(\mathcal{D}_{N}(a) \smile \mathcal{D}_{N}(b)\right) \cap[N]=\mathcal{D}_{N}(a) \cap\left(\mathcal{D}_{N}(b) \cap[N]\right) \\
& =\mathcal{D}_{N}(a) \cap b .
\end{aligned}
$$

Let $f: N^{\prime} \rightarrow N$ be a smooth map between closed, connected, oriented manifolds of respective fundamental classes $\left[N^{\prime}\right] \in H_{n^{\prime}}\left(N^{\prime}\right)$ and $[N] \in H_{n}(N)$. The homological transfer ([3, Section 11 of Chapter VII]) of $f$ is

$f_{!}=(-1)^{j\left(n^{\prime}-n\right)} \mathcal{D}_{N^{\prime}}^{-1} \circ f^{*} \circ \mathcal{D}_{N}: H_{j}(N) \rightarrow H^{n-j}(N) \rightarrow H^{n-j}\left(N^{\prime}\right) \rightarrow H_{n^{\prime}-n+j}\left(N^{\prime}\right)$.

In the case of the diagonal map $\Delta: M \rightarrow M \times M$ of a closed, connected, oriented manifold of fundamental class $[M] \in H_{m}(M)$, the two previous notions are related by

$$
\begin{aligned}
\Delta_{!}(a \times b) & =(-1)^{j m}\left(\mathcal{D}_{M}^{-1} \circ \Delta^{*} \circ \mathcal{D}_{M \times M}\right)(a \times b) \\
& =(-1)^{m(m+|a|)} \mathcal{D}_{M}^{-1}\left(\Delta^{*}\left(\mathcal{D}_{M}(a) \times \mathcal{D}_{M}(b)\right)\right) \\
& =(-1)^{m(m+|a|)}\left(\mathcal{D}_{M}(a) \smile \mathcal{D}_{M}(b)\right) \cap[M] \\
& =(-1)^{m(m-|a|)} a \pitchfork b .
\end{aligned}
$$

Given an oriented vector bundle of rank $k, \xi: E(\xi) \rightarrow N$, over $N$, there are two associated bundles over $N$ : the disk bundle, $D(\xi)$, consisting of all $(x, v) \in E(\xi)$ with $\|v\| \leq 1$, and the sphere bundle, $S(\xi)$, consisting of all $(x, v) \in E(\xi)$ with $\|v\|=1$.

Definition 1.1. The cofiber of the inclusion $S(\xi) \hookrightarrow D(\xi)$ is the Thom space of the bundle $\xi$, denoted $\operatorname{Th}(\xi)$. We also write

$$
\operatorname{Th}(\xi)=D(\xi) / S(\xi) \text {. }
$$


In [14, Thom proves the existence of an isomorphism

$$
H_{*}(N) \cong H_{*+k}(D(\xi), S(\xi)) \cong H_{*+k}(\operatorname{Th}(\xi)),
$$

induced by the cap product with a cohomology class, called the Thom class, $U \in H^{k}(D(\xi), S(\xi))$. Below we will give a more explicit description of the Thom isomorphism in terms of intersection product in the case of the tangent bundle.

In order to do that, we must relate the tangent space $T M$ of an $m$-manifold $M^{m}$ to the configuration space of two points in $M$. Recall that the configuration space of $k$ points in a manifold $M$ is defined by

$$
F(M, k)=\left\{\left(x_{1}, \ldots, x_{k}\right) \mid x_{i} \neq x_{j} \text {, for } i \neq j\right\} .
$$

The case $k=2$ allows a nicer description as the complement of the diagonal map $\Delta: M \rightarrow M \times M$ (see [5, 7]). Indeed, by identifying the disk bundle of the normal bundle $\nu_{\Delta}$ of the embedding $\Delta$ with a closed tubular neighborhood $T \simeq M$ in $M \times M$, we see that the left-hand square of the next diagram is a (homotopy) pushout diagram

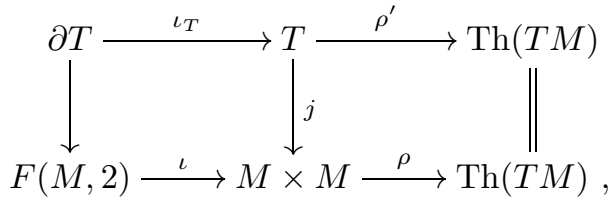

where $j: T \rightarrow M \times M$ is homotopic to the diagonal map $\Delta$ and the maps $\rho$ and $\rho^{\prime}$ are defined as follows. Because $T \cong D\left(\nu_{\Delta}\right)$ and $\partial T \cong S\left(\nu_{\Delta}\right)$, the map $\iota_{T}$ has cofiber $\operatorname{Th}\left(\nu_{\Delta}\right)$. Because the diagram is a pushout, the bottom row, $\iota$, also has cofiber $\operatorname{Th}\left(\nu_{\Delta}\right)$ up to homotopy type. Finally, it is well known that the normal bundle $\nu_{\Delta}$ is in fact isomorphic to the tangent bundle of $M$ (see [9]). Hence, both cofibers are really $\operatorname{Th}(T M)$, as indicated in the diagram.

Proposition 1.2. The map induced in homology by the projection to the cofiber, $\rho: M \times M \rightarrow \operatorname{Th}(T M)$, is given by

$$
\mathrm{Th}_{*} \circ \rho_{*}(a \times b)=(-1)^{m(m-\mid a])} a \pitchfork b=\Delta_{!}(a \times b) .
$$

Since $[M] \pitchfork a=a \pitchfork[M]=a$, the map $\rho_{*}$ is surjective in homology.

Proof. The fundamental class $[M] \times[M] \in H_{2 m}(M \times M)$ of the product induces fundamental classes $\xi_{M} \in H_{2 m}\left(M \times M,(M \times M) \backslash \Delta_{M}\right)$ and $[T] \in H_{2 m}(T, \partial T)$ by excision. These classes produce isomorphisms

$$
-\cap[T]: H^{2 m-k}(T) \rightarrow H_{k}(T, \partial T)
$$

and

$$
-\cap\left[\xi_{M}\right]: H^{2 m-k}(M) \rightarrow H_{k}\left(M \times M,(M \times M) \backslash \Delta_{M}\right),
$$

whose inverse maps are respectively denoted $\mathcal{D}_{T}$ and $\mathcal{D}_{(M \times M) \backslash \Delta_{M}}$. These isomorphisms are compatible with the long exact sequences associated to the pair $\left(M \times M, \Delta_{M}\right)$ (see [1, Corollary 8.4, page 352]); that is, the following diagram is commutative:

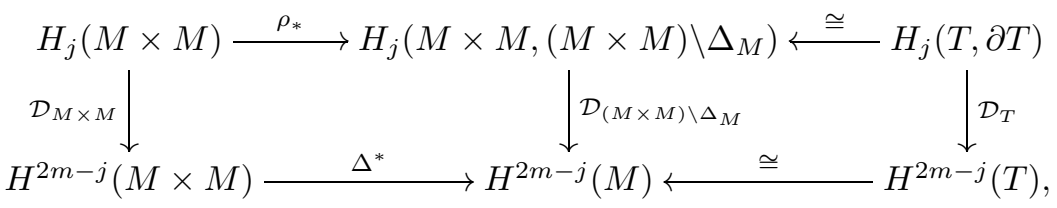


where the right-hand horizontal maps are isomorphisms by excision.

With the preliminaries above, we can now describe the Thom isomorphism. Denote by $i: M \rightarrow T$ the canonical inclusion. The Thom class $U \in H^{m}(T, \partial T)$, defined by

induces isomorphisms

$$
U \cap[T]=i_{*}([M]) \in H_{m}(T, \partial T),
$$

$$
\mathrm{Th}_{*}(-)=U \cap-: H_{j+m}(T, \partial T) \rightarrow H_{j}(T)
$$

and

$$
\mathrm{Th}^{*}(-)=U \smile-: H^{j}(T) \rightarrow H^{j+m}(T, \partial T) .
$$

These isomorphisms are compatible with taking the cap product with $[T]$, as we can see by computing, for $x \in H^{2 m-j}(T)$,

$$
\left(\mathrm{Th}_{*}\right) \circ(-\cap[T])(x)=U \cap(x \cap[T])=(U \smile x) \cap[T]=(-\cap[T]) \circ \mathrm{Th}^{*}(x) .
$$

We therefore have that the following diagram is commutative:

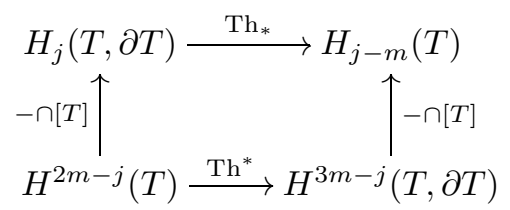

Now, adjoining this diagram to the one above we obtain a commutative diagram,

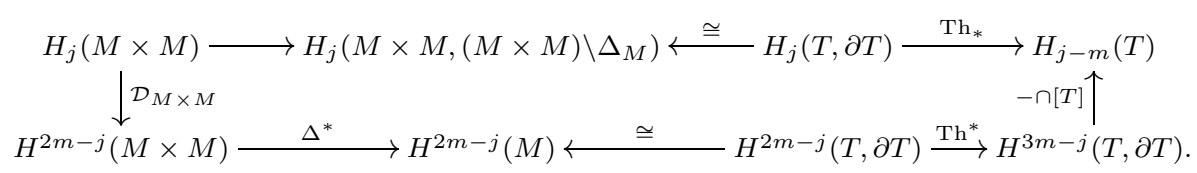

The composition of the top line is exactly $\mathrm{Th}_{*} \circ \rho_{*}$. We proceed down, across and up and obtain

$$
\begin{aligned}
a \times b & \mapsto(-1)^{m(m-[b])} \mathcal{D}_{M}(a) \times \mathcal{D}_{M}(b) \mapsto(-1)^{m(m-[b])} \mathcal{D}_{M}(a) \smile \mathcal{D}_{M}(b) \\
& \mapsto(-1)^{m(m-[b])} U \smile \mathcal{D}_{M}(a) \smile \mathcal{D}_{M}(b)=(-1)^{m(m-|a|)} \mathcal{D}_{M}(a) \smile \mathcal{D}_{M}(b) \smile U \\
& \mapsto(-1)^{m(m-|a|)}\left(\mathcal{D}_{M}(a) \smile \mathcal{D}_{M}(b) \smile U\right) \cap[T] \\
& =(-1)^{m(m-|a|)}\left(\mathcal{D}_{M}(a) \smile \mathcal{D}_{M}(b)\right) \cap i_{*}([M]) \\
& =(-1)^{m(m-|a|)} a \pitchfork b=\Delta_{!}(a \times b) .
\end{aligned}
$$

\section{Rational homotopy type of Thom sPaCES}

The commutative differential graded algebra (henceforth cdga) version of rational homotopy theory, introduced by Sullivan ([12]), constructs algebraic models for spaces of the form $(\wedge V, d)$, where $\wedge V$ is the algebra freely generated by the graded vector space $V$ and $d$ is a graded algebra differential. These are delineated in, for example, 4, 5], where the statements that follow are elaborated on. When the differential is decomposable (i.e., $d(v) \in \wedge^{+} V \cdot \wedge^{+} V$ ), then the algebraic model is called minimal and has, within it, knowledge of the complete rational homotopy type of the space it corresponds to. For example, if the finite-type nilpotent space $X$ has algebraic minimal model $(\wedge V, d)$, then we know in particular that $H^{*}(X ; \mathbb{Q}) \cong$ $H^{*}(\wedge V, d)$ and $V \cong \operatorname{Hom}\left(\pi_{*}(X), \mathbb{Q}\right)$. 
Now, in order to understand the rational homotopy type of a Thom space $\operatorname{Th}(\xi)=D(\xi) / S(\xi)$, we just need to write down a model of the inclusion of the sphere bundle into the disk bundle, $S(\xi) \hookrightarrow D(\xi)$, as a cofibration. For this, we recall the so-called surjective model for a map $f: X \rightarrow Y$. Let $(\wedge W, d)$ and $(\wedge V, d)$ be models for $Y$ and $X$ respectively and consider the cdga morphism $F:(\wedge W, d) \rightarrow(\wedge V, d)$ guaranteed by the general theory. Then, after tensoring with the acyclic cdga $\wedge(V, d V)$, we define a cdga morphism

$$
\hat{F}:(\wedge W \otimes \wedge(V, d V), d) \rightarrow\left(\wedge V, d^{\prime}\right)
$$

by restricting to $F$ on $\wedge W, v \mapsto v$ and $(d v) \mapsto d(v)$. We have the following; see [4].

Theorem 2.1. Let $\hat{F}:(\wedge W \otimes \wedge(V, d V), d) \rightarrow\left(\wedge V, d^{\prime}\right)$ be the surjective model of the map $f: X \rightarrow Y$. Then the kernel, $\operatorname{Ker}(\hat{F})$, together with the field $\mathbb{Q}$ in degree 0 , is a model (usually non-free) for the cofiber of $f$.

Example 2.2. We illustrate this technique with the classical and well-known example of the Hopf fibration, $h: S^{7} \rightarrow S^{4}$. A surjective model of it is given by

$$
\hat{H}:\left(\wedge(e, a) \otimes \wedge(\bar{e}, z), d e=0, d a=e^{2}, d \bar{e}=z, d z=0\right) \rightarrow(\wedge(\bar{e}), d \bar{e}=0)
$$

with $|e|=4,|\bar{e}|=|a|=7,|z|=8, e \mapsto 0, a \mapsto \bar{e}, \bar{e} \mapsto \bar{e}$ and $z \mapsto 0$. The kernel is given by $\wedge(e, a, \bar{e}, z) \cdot \wedge^{+}(e \oplus z \oplus(\bar{e}-a))$. This is the ideal generated by $e, z$ and $\bar{e}-a$. First note that we can change generators by taking $\hat{e}=\bar{e}-a$. Then quotienting by the acyclic ideal $(\hat{e}, z)($ since $d \hat{e}=z)$, we get a quasi-isomorphism

$$
\wedge(e, a, \hat{e}, z) \cdot \wedge^{+}(e \oplus z \oplus \hat{e}) \stackrel{\simeq}{\rightarrow} \wedge(e, a) \cdot \wedge^{+}(e),
$$

with $d(e a)=e^{3}$. Note that $a$ itself is not in this algebra, so $e^{2}$ is a non-trivial cocycle. Therefore, the cohomology of the cofiber is the truncated polynomial algebra $\mathbb{Q}[e] /\left(e^{3}\right)$. Denote by $i_{4}=\mathrm{id}: S^{4} \rightarrow S^{4}$ the identity map. A minimal model for $\left(\mathbb{Q}[e] /\left(e^{3}\right), 0\right)$ is $\left(\wedge(e, y), d e=0, d y=e^{3}\right)$, with $|y|=11$, and, as expected, the corresponding space is $\mathbb{H P}(2)=S^{4} \cup_{\left[i_{4}, i_{4}\right]} e^{8}$.

Let $N$ be a closed, oriented, nilpotent manifold, of finite type, and let $\xi: E(\xi) \rightarrow$ $N$ be an oriented vector bundle of rank $k$ with associated disk bundle $D(\xi)$ and sphere bundle $S(\xi)$. As the space $D(\xi)$ has the homotopy type of $N$, the Thom space $\operatorname{Th}(\xi)$ is homotopically equivalent to the homotopy cofiber of the fibration $S(\xi) \rightarrow N$. (For instance, if $\xi$ is a trivial bundle, the Thom space has the homotopy type of $\left(N \times D^{k}\right) /\left(N \times S^{k-1}\right)=\Sigma^{k}\left(N_{+}\right)=\Sigma^{k} N \vee S^{k}$.)

Models for sphere bundles come in two forms depending on the parity of the bundle rank; see [4, Example 4, page 202].

Proposition 2.3. Let $\xi: E(\xi) \rightarrow N$ be an oriented vector bundle of rank $k$ and let $(\wedge V, d)$ be a model for $N$.

(1) If $k$ is even, then a model for $S(\xi)$ is given by $(\wedge V \otimes \wedge(e), d)$, with $|e|=k-1$, $\left.d\right|_{V}=d$ and $d e=\chi$, where $\chi$ is a representative for the Euler class of $\xi$.

(2) If $k$ is odd, then a model for $S(\xi)$ is given by $(\wedge V \otimes \wedge(e, a), d)$, with $|e|=$ $k-1,|a|=2 k-3,\left.d\right|_{V}=d$, de $=0$ and $d a=e^{2}+p$, where $p$ is a representative of the $(k-1) / 2$-th rational Pontryagin class of $\xi$ in $\wedge V$.

Recall that the (rational) Euler class of an odd-dimensional vector bundle is always zero; see [9, Property 9.4].

The rational homotopy type of $\operatorname{MSO}(n), \mathrm{MO}(n), \mathrm{MU}(n)$, with $n$ odd, was studied by Burlet ([2]); see also [10] for the case of a closed connected subgroup of $\operatorname{MSO}(n)$. 
We now classify Thom spaces of bundles up to rational homotopy type and specify the particular case of the tangent bundle in Proposition 3.1

Theorem 2.4. Let $\xi: E(\xi) \rightarrow N$ be an oriented vector bundle of rank $k$. Then there are two cases.

(1) If the rational Euler class of $\xi$ is zero, then

$$
\operatorname{Th}(\xi) \simeq_{\mathbb{Q}} \Sigma^{k} N \vee S^{k} .
$$

(2) Let $(\wedge V, d)$ be a minimal model for $N$. If the rational Euler class of $\xi$ is non-zero with representative $\chi \in \wedge V$, then a model for $\operatorname{Th}(\xi)$ is given by

$$
(\mathbb{Q} \oplus(\wedge V \otimes z), d),
$$

with $|z|=k, z^{2}=z \chi$ and general product $(a \otimes z) \cdot(b \otimes z)=a b \chi \otimes z$, inducing the algebra structure on $H^{*}(\operatorname{Th}(\xi) ; \mathbb{Q}) \cong \mathbb{Q} \oplus\left(H^{*}(N) \otimes z\right)$.

Proof. Let $(\wedge V, d)$ be a model for $N$.

(1) Suppose $\chi=0$. We first consider an even-dimensional vector bundle. We are in the first case of Proposition 2.3, so a model of $S(\xi) \rightarrow N$ is given by $(\wedge V, d) \rightarrow$ $(\wedge V \otimes \wedge(e), d),|e|=k-1,\left.d\right|_{V}=d, d e=0$. By Theorem 2.1, the kernel of a surjective model, together with the field in degree 0 , is a model of the homotopy cofiber, $\operatorname{Th}(\xi)$. Therefore, we build a surjective model starting from the previous model, as:

$$
\varphi:(\wedge V \otimes \wedge(e, z), D) \rightarrow(\wedge V \otimes \wedge(e), d),
$$

where $|e|=k-1,|z|=k,\left.D\right|_{V}=d, D e=z, D z=0, \varphi(z)=0$ and $\varphi(e)=e$. The kernel of $\varphi$ is given by

$$
(\wedge V \otimes \wedge(e)) \cdot \wedge^{+}(z) .
$$

Because $D(e z)=z^{2}$, we can quotient the kernel by the acyclic ideal generated by $\left(e z, z^{2}\right)$ and get a quasi-isomorphism between this kernel and $(\wedge V \otimes z, d)$ with $z^{2}=0$. As there are no products in this last model, we can map $H^{+}\left(\Sigma^{k} N\right) \oplus H^{+}\left(S^{k}\right)$ to $(\wedge V \otimes z, d)$ by a cdga map. This proves that the Thom space, $\operatorname{Th}(\xi)$, has the rational homotopy type of $\Sigma^{k} N \vee S^{k}$.

We now consider the case of an odd-dimensional vector bundle. As before, starting from a model of the sphere bundle given by Proposition 2.3 , we construct a surjective model of $S(\xi) \rightarrow N$. For that, consider

$$
\varphi:(\wedge V \otimes \wedge(e, z, a, y), D) \rightarrow(\wedge V \otimes \wedge(e, a), d),
$$

where $|e|=k-1,|a|=2 k-3,\left.d\right|_{V}=d, d e=0, d a=e^{2}+p,|z|=k,|y|=2 k-2$, $\left.D\right|_{V}=d, D e=z, D z=0, D a=e^{2}+p+y, D y=-2 e z, \varphi(e)=e, \varphi(a)=a$ and $\varphi(z)=\varphi(y)=0$. A verification shows that $D^{2}=0$. The canonical inclusion, $(\wedge V, d) \rightarrow(\wedge V \otimes \wedge(e, z, a, y), D)$, can be filtered by the degree in $\wedge V$. The first term of the associated spectral sequence is

$$
\wedge V \rightarrow \wedge V \otimes H^{*}(\wedge(e, z, a, y), \bar{D})
$$

with $\bar{D} y=-2 e z, \bar{D} z=0, \bar{D} a=e^{2}+y, \bar{D} e=z$. By considering the linear part of $\bar{D}$, we have $H^{*}((\wedge(e, a, z, y), \bar{D}))=\mathbb{Q}$ and we may conclude that $\varphi$ is a surjective model of $S(\xi) \rightarrow N$. Observe also that the tensor product of $\varphi$ with $\mathbb{Q}$ over $\wedge V$ gives a short exact sequence,

$$
0 \rightarrow K \rightarrow(\wedge(e, a, z, y), \bar{D}) \stackrel{\mathbb{Q} \otimes_{\wedge} \varphi}{\longrightarrow}(\wedge(e, a), \bar{d}) \rightarrow 0,
$$


with $K=\wedge(e, a) \otimes \wedge^{+}(z, y)$. From the long exact sequence associated to this short exact sequence, we deduce that the canonical inclusion, $\wedge^{+} z \rightarrow K$, is a quasiisomorphism.

We now have to study the kernel of the surjective model, $\varphi$, which is equal to

$$
\wedge V \otimes \wedge(e, a) \otimes \wedge^{+}(z, y) .
$$

Consider the following canonical inclusion, with the induced differentials:

$$
\wedge V \otimes \wedge^{+} z \rightarrow \operatorname{ker} \varphi=\wedge V \otimes \wedge(e, a) \otimes \wedge^{+}(z, y) .
$$

As before, we filter each term by the degree in $\wedge V$ and get a morphism of spectral sequences whose first stage is

$$
\wedge V \otimes \wedge^{+} z \rightarrow \wedge V \otimes H^{*}\left(\wedge(e, a) \otimes \wedge^{+}(z, y), \bar{D}\right)=\wedge V \otimes \wedge^{+} z .
$$

(The equality comes from the previous determination of $H^{*}(K)=$ $H^{*}\left(\wedge(e, a) \otimes \wedge^{+}(z, y)\right)=\wedge^{+} z$. $)$ Since we have an isomorphism at the $E_{1}$-level, this canonical inclusion is a quasi-isomorphism. Thus, since the cdga $\left(\wedge V \otimes \wedge^{+} z, D\right)$ is connected to the kernel of $\varphi$ by a quasi-isomorphism, we deduce from Theorem 2.1 that this cdga is a (non-free) model of $\operatorname{Th}(\xi)$. The same argument as in the first case ends the proof.

(2) The remaining case is $k$ even with a non-trivial Euler class. As previously, we deduce, from Proposition 2.3, a surjective model of $S(\xi) \rightarrow N$ as

$$
\varphi:(\wedge V \otimes \wedge(e, z), D) \rightarrow(\wedge V \otimes \wedge(e), d),
$$

where $|e|=|e|=k-1,|z|=k,\left.d\right|_{V}=\left.D\right|_{V}=d, d e=\chi, D e=-z+\chi, D z=0$, $\varphi(e)=e$ and $\varphi(z)=0$. The kernel of $\varphi$ is given by

$$
(\wedge V \otimes \wedge(e)) \otimes \wedge^{+}(z) .
$$

We quotient by the acyclic ideal generated by $\left(e z, D(e z)=-z^{2}+\chi z\right)$ and get a quasi-isomorphism between this kernel and $(\wedge V \otimes z, D)$, with the product $z^{2}=\chi z$. More generally, the product rule of $(\wedge V \otimes z, D)$ is given by $(a \otimes z) \cdot(b \otimes z)=a b \chi \otimes z$, as stated.

The model for $\operatorname{Th}(\xi)$ displays the Thom class explicitly as $z$ and, moreover, the structure of the model as the minimal model $(\wedge V, d)$ of $N$ tensored with $z$ is precisely the Thom isomorphism. But notice that this is at the level of models rather than simply for cohomology.

Our description of a model for the Thom space in fact holds for any spherical fibration and immediately gives a result of Marcum and Randall [8]. Since the fiber $S^{k-1}$ maps to a point with cofiber $S^{k}$, we have an induced map on cofibers,

$$
\mathrm{MU}: S^{k} \rightarrow \operatorname{Th}(\xi),
$$

called the homotopy Thom class.

Corollary 2.5. If $k$ is even and the rational Euler class is zero, then the Whitehead product $[\mathrm{MU}, \mathrm{MU}] \in \pi_{2 k-1}(\mathrm{Th}(\xi))$ is not trivial.

Proof. By Theorem 2.4. we have $\operatorname{Th}(\xi) \simeq_{\mathbb{Q}} \Sigma^{k} N \vee S^{k}$ and the homotopy Thom class is simply the inclusion of the sphere $S^{k}$ into the wedge. Hence, [MU, MU] $=\left[i_{k}, i_{k}\right]$, where $i_{k}$ is the identity map of $S^{k}$ and, classically, $\left[i_{k}, i_{k}\right] \neq 0$ for $k$ even. 


\section{Lie-Model of the CANONICAL MAP $M \times M \rightarrow \operatorname{Th}(T M)$}

In the case of the tangent bundle of a manifold $M$, Theorem 2.4 takes the following more explicit form.

Proposition 3.1. Let $\xi=T M$ be the tangent bundle of a closed, connected, oriented, nilpotent, $m$-dimensional manifold $M$ of fundamental class $[M] \in H_{n}(M)$.

(1) If the rational Euler class of $T M$ is zero, then

$$
\operatorname{Th}(T M) \simeq_{\mathbb{Q}} \Sigma^{m} M \vee S^{m} .
$$

(2) If the rational Euler class of $T M$ is non-zero, then

$$
\begin{aligned}
& \operatorname{Th}(T M) \simeq_{\mathbb{Q}} \Sigma^{m}(M \backslash\{p\}) \vee\left(S^{m} \cup_{\left[i_{m}, i_{m}\right]} e^{2 m}\right) \simeq_{\mathbb{Q}} \Sigma^{m}(M \backslash\{p\}) \vee \operatorname{Th}\left(T S^{m}\right), \\
& \text { where }\left[i_{m}, i_{m}\right] \text { is the self-Whitehead product of the identity map } i_{m} \text { of } S^{m} \\
& \text { and } T S^{m} \text { is the tangent bundle of } S^{m} .
\end{aligned}
$$

Proof. Only the second part requires proof, so we take $m$ even and $\chi \neq 0$. For the tangent bundle, the Euler class $\chi$ is the product of the top cohomology class by the Euler characteristic, i.e., $\chi=\chi(M) \omega$, where $\omega$ is the cohomology class, Kronecker dual to the fundamental class $[M]$; see [1, Chapter VI, Corollary 12.5].

The minimal model $(\wedge V, d)$ of $M$ is quasi-isomorphic to the cdga

$$
(A, d)=\left(\wedge V /\left((\wedge V)^{\geq m+1} \oplus\left(Z V^{c}\right)\right), d\right),
$$

with the induced differential, where $Z V^{c}$ denotes the complement of the cocycles in degree $m$. Replacing $(\wedge V, d)$ by $(A, d)$ in the model of Theorem 2.4, we get as the model of the Thom space $(\mathbb{Q} \oplus(A \otimes z), d)$ with product $(a \otimes z) \cdot(b \otimes z)=a b \chi \otimes z$. This means, for degree reasons, that there is only one non-trivial product,

$$
(1 \otimes z) \cdot(1 \otimes z)=\chi(M) \omega \otimes z .
$$

This Thom space model can also be written as

$$
A \otimes z=(S \otimes z) \oplus(1 \otimes z) \oplus(\omega \otimes z),
$$

where $S$ is a supplementary subspace, in $A^{+}$, of the vector subspace $\langle\omega\rangle$ generated by $\omega$, i.e., $S \oplus\langle\omega\rangle=A^{+}$. In this direct sum, notice that the first term has no non-trivial products and there are no cross products for degree reasons. The two last terms are a model for a space with rational cohomology in degrees $m$ and $2 m$ with cup product $(1 \otimes z)^{2}=\omega \otimes z$. This is a space with the same rational homotopy type as $S^{m} \cup_{\left[i_{m}, i_{m}\right]} e^{2 m}$. To see that this is the Thom space of $T S^{m}$, we have only to observe that, in the case $M=S^{m}$, the corresponding cdga $A \otimes z$ reduces to $(1 \otimes z) \oplus(\omega \otimes z)$.

As Quillen shows in [11, differential Lie algebras give an algebraic approach to rational homotopy theory for simply-connected spaces. For the concrete constructions of Lie-models of products, fibrations and cofibrations, we refer the reader to [13. In particular, the minimal Lie-model of a simply-connected space $X$ has the form $\mathcal{L}_{X}=(\mathbb{L}(V), \partial)$, where $V=s^{-1}\left(\bar{H}_{*}(X)\right)$ is a desuspension of the reduced homology and $\mathbb{L}(V)$ denotes the free graded Lie algebra on $V$. The product $X \times X$ has a minimal Lie-model $\mathcal{L}_{X \times X}=\left(\mathbb{L}\left(s^{-1} \bar{H}_{*}(X \times X)\right)\right.$, $\left.\partial\right)$ that can be built from the minimal Lie-model $\mathcal{L}_{X}$ of $X$. Note also that a continuous map $f: X \rightarrow Y$ has a Lie-model $\varphi:\left(\mathbb{L}\left(s^{-1} \bar{H}_{*}(X)\right), \partial\right) \rightarrow\left(\mathbb{L}\left(s^{-1} \bar{H}_{*}(Y)\right), \partial\right)$, and the map induced by $\varphi$ between the indecomposables of the Lie algebras is the map $s^{-1} \bar{H}_{*}(f)$. 
From Proposition 1.2, we know that the map induced in homology by the projection $\rho: M \times M \rightarrow \operatorname{Th}(T M)$ is given by $\rho_{*}(a \times b)=(-1)^{|a|}(a \pitchfork b) \otimes[M]$.

Theorem 3.2. Let $M$ be a closed, oriented, simply-connected manifold with fundamental class $[M] \in H_{n}(M)$. The minimal Lie-model of $\rho: M \times M \rightarrow \operatorname{Th}(T M)$ is given by

- $\left(\mathbb{L}\left(s^{-1} \bar{H}_{*}(M \times M)\right), \partial\right) \stackrel{\mathbb{L}\left(s^{-1} \rho_{*}\right)}{\longrightarrow}\left(\mathbb{L}\left(s^{-1} H_{*}(M) \otimes[M]\right), 0\right)$, if $M$ is odddimensional,

- $\left(\mathbb{L}\left(s^{-1} \bar{H}_{*}(M \times M)\right), \partial\right) \stackrel{\mathbb{L}\left(s^{-1} \rho_{*}\right)}{\longrightarrow}\left(\mathbb{L}\left(s^{-1} H_{*}(M) \otimes[M]\right), \bar{\partial}\right)$, if $M$ is evendimensional and where the differential $\bar{\partial}$ is equal to zero on all generators except for

$$
\bar{\partial}\left(s^{-1}[M] \otimes[M]\right)=\left[s^{-1} 1 \otimes[M], s^{-1} 1 \otimes[M]\right] .
$$

Because we have a cofiber sequence $F(M, 2) \hookrightarrow M \times M \stackrel{\rho}{\rightarrow} \operatorname{Th}(T M)$, general properties of Lie models lead us to the following conjecture. In Remark 4.3, we observe that the conjecture holds for some fundamental examples.

Conjecture 3.3. With the notation of Theorem 3.2, we conjecture that a model of $F(M, 2)$ is given by $\left(\mathbb{L}\left(\operatorname{ker} s^{-1} \rho_{*}\right), \partial\right)$, where the differential $\partial$ is induced by the differential of the Lie-model of $M \times M$.

Proof of Theorem 3.2. As the case $M=S^{2}$ is detailed in Example 4.1, we may suppose $m \geq 3$.

If $M$ is odd-dimensional, the Thom space $\operatorname{Th}(T M)$ is a suspension and has minimal Lie-model $\mathcal{L}_{\mathrm{Th}(T M)}=\left(\mathbb{L}\left(s^{-1} H_{*}(M) \otimes[M]\right), 0\right)$; see Proposition 3.1. If $M$ is even-dimensional, the Thom space $\operatorname{Th}(T M)$ is a wedge of a suspension and a two-cell space whose attaching map is a Whitehead bracket; see Proposition 3.1. Thus it has for minimal Lie-model the differential Lie algebra

$$
\mathcal{L}_{\mathrm{Th}(T M)}=\left(\mathbb{L}\left(s^{-1} H_{*}(M) \otimes[M]\right), \bar{\partial}\right)
$$

described in the statement. The result is therefore a direct consequence of the following claim.

Claim. Any linear map

$$
s^{-1} \bar{H}_{*}(M \times M) \longrightarrow \mathbb{L}^{\geq 2}\left(s^{-1} H_{*}(M) \otimes[M]\right)
$$

is trivial, where $\mathbb{L}^{\geq 2}$ denotes the subspace of the free Lie algebra generated by brackets of size greater than or equal to 2 .

In order to prove this, we have only to determine the respective degrees. First, we observe that the space $s^{-1} \bar{H}_{*}(M \times M)$ is such that:

- the subspace of elements of degree $2 m-1$ is of dimension 1 ,

- the other elements have degrees less than or equal to $2 m-3$.

Furthermore, the space $s^{-1} H_{*}(M) \otimes[M]$ is such that:

- the subspace of elements of degree $m-1$ is of dimension 1 ,

- the other elements have degrees greater than or equal to $m+1$.

If $m$ is odd, the elements of

- $\mathbb{L}^{2}\left(s^{-1} H_{*}(M) \otimes[M]\right)$ are of degree greater than or equal to $2 m$,

- $\mathbb{L}^{k}\left(s^{-1} H_{*}(M) \otimes[M]\right)$ are of degree greater than or equal to $(k-1)(m-1)+$ $m+1=k m-k+2$. 
If $2 m-1=k m-k+2$, then

$$
m=\frac{k-3}{k-2}=\frac{-1}{k-2}+1,
$$

which is impossible.

If $m$ is even, the only difference is that $\mathbb{L}^{2}\left(s^{-1} H_{*}(M) \otimes[M]\right)$ has elements of degree $2 m-2$, but there is no element of this degree in the domain of the map. Thus the result follows.

\section{EXAmples}

Example 4.1 (Spheres). We shall consider the odd- and even-dimensional cases separately.

(1) Let $M=S^{m}$ with $m$ odd. Then the Thom space is $\operatorname{Th}(T M)=S^{m} \vee S^{2 m}$ and the Lie-model of $\rho: M \times M \rightarrow \operatorname{Th}(T M)$ is given by

$$
\mathbb{L}(y, v, s(y v)) \rightarrow \mathbb{L}(z, e),
$$

where $\partial y=0=\partial v, \partial s(y v)=[y, v], \partial z=0=\partial e, \rho_{*}(y)=\rho_{*}(v)=z$ and $\rho_{*}(s(y v))=[T]$ (where $[T]$ is the fundamental class of $(T, \partial T)$ as in Proposition 1.2). To see how the values for $\rho_{*}$ are determined, consider $\rho_{*}(s(y v))$. This class in the homology of $S^{m} \times S^{m}$ is represented by $[M] \times[M]$. Clearly, $[M] \pitchfork[M]=[M]$ and the inverse of the Thom isomorphism applied to $[M]$ is $[T]$ by the basic formula $U \cap[T]=[M]$, where $U \in H^{m}(T, \partial T)$ is the Thom class. Note that $\rho_{*}(\partial s(y v))=$ $[z, z]=0=\partial e$ since $|z|=m-1$ is even.

(2) Let $M=S^{m}$ with $m$ even. Then the Thom space is $\operatorname{Th}(T M)=S^{m} \cup_{[z, z]} e^{2 m}$ and the Lie-model of $\rho: M \times M \rightarrow \operatorname{Th}(T M)$ is given by

$$
\mathbb{L}(y, v, s(y v)) \rightarrow \mathbb{L}(z, e),
$$

where $\partial y=0=\partial v, \partial s(y v)=[y, v], \partial z=0, \partial e=\frac{1}{2}[z, z], \rho_{*}(y)=\rho_{*}(v)=z$ and $\rho_{*}(s(y v))=[T]$. Here, the $\frac{1}{2}$ in the differential for $e$ arises from the diagonal in the associated coalgebra. Now, however, we have $\rho_{*}(\partial s(y v))=[z, z] \neq 0$, so we require the correct $\partial[T]$. Here is where we bring in the geometry of the Thom isomorphism. Recall that in $H^{*}(T, \partial T)$, we have $U=\chi$ (where $\chi$ is the pullback of the Euler class) and

$$
U \smile U=\chi \smile U=\chi(M) \omega_{M} \smile U=2 \omega_{T},
$$

where $\omega_{M}, \omega_{T}$ are top cohomology classes Kronecker dual to the respective fundamental classes $[M],[T]$. Again the basic formula $U \cap[T]=[M]$ gives

$$
\left\langle\omega_{M} \smile U,[T]\right\rangle=\left\langle\omega_{M}, U \cap[T]\right\rangle=\left\langle\omega_{M},[M]\right\rangle=1,
$$

so we see that $\omega_{M} \smile U=\omega_{T}$. This calculation says that the Kronecker dual of $U \smile U$ has two descriptions: (1) $e$ with $\partial e=\frac{1}{2}[z, z]$; (2) $2 e=[T]$, since $e$ is Kronecker dual to $U \smile U=2 \omega_{M} \smile U$ which is Kronecker dual to $\frac{1}{2}[T]$. Thus, we have

$$
\rho_{*}(\partial s(y v))=\rho_{*}([y, v])=[z, z]=2\left(\frac{1}{2}\right)[z, z]=\partial(2 e)=\partial([T]),
$$

and $\rho_{*}$ is well defined.

Example 4.2. If $M=\mathbb{C P}^{2}$, the Thom space is

$$
\operatorname{Th}(T M)=\Sigma^{m}\left(\mathbb{C P}^{2} \backslash\{p\}\right) \vee S^{4} \cup_{[z, z]} e^{8}
$$


and the Lie-model of $\rho: M \times M \rightarrow \operatorname{Th}(T M)$ is given by

$$
\mathbb{L}(x, y, u, v, s(x u), s(x v), s(y u), s(y v)) \rightarrow \mathbb{L}(z, w, e),
$$

where $|x|=|u|=1,|y|=|v|=|s(x u)|=|z|=3,|s(x v)|=|s(y u)|=|w|=5$, $|s(y v)|=|e|=7, \partial x=0=\partial u, \partial y=\frac{1}{2}[x, x], \partial v=\frac{1}{2}[u, u]$, and

$$
\begin{aligned}
& \partial s(x u)=[x, u] \\
& \partial s(x v)=[x, v]+[u, s(x u)], \\
& \partial s(y u)=[y, u]+[x, s(x u)], \\
& \partial s(y v)=[y, v]+[x, s(x v)]+[u, s(y u)]+\frac{1}{2}[s(x u), s(x u)],
\end{aligned}
$$

with $\partial z=0=\partial w, \partial e=\frac{1}{2}[z, z]$. The map is given by

$$
\begin{aligned}
\rho_{*}(x) & =\rho_{*}(u)=0, \\
\rho_{*}(y) & =\rho_{*}(v)=\rho_{*}(s(x u))=z, \\
\rho_{*}(s(x v)) & =\rho_{*}(s(y u))=w, \\
\rho_{*}(s(y v)) & =[T] .
\end{aligned}
$$

Now here, $\chi(M)=3$, so $[T]=3 e$ and we have

$$
\rho_{*}(\partial s(y v))=\frac{3}{2}[z, z]=3 \cdot \frac{1}{2}[z, z]=\partial(3 e)=\partial([T]) .
$$

Remark 4.3. In the case $M=S^{m}$, the Lie differential algebra $\left(\mathbb{L}\left(\operatorname{ker} \rho_{*}\right), \partial\right)$ is isomorphic to $(\mathbb{L}(\alpha), 0)$, with $|\alpha|=m-1$. It coincides with the integral description $F(M, 2) \simeq S^{m}$.

In the case $M=\mathbb{C P}^{2}$, we obtain, for $\left(\mathbb{L}\left(\operatorname{ker} \rho_{*}\right), \partial\right)$,

$$
\mathbb{L}(x, u, \bar{y}, \bar{v}, t),
$$

where $|x|=|u|=1,|\bar{y}|=|\bar{v}|=3,|t|=5, \bar{y}=y-s(x u), \bar{v}=v-s(x u)$ and $t=s(x v)-s(y u)$. Thus,

$$
\begin{aligned}
\partial x & =\partial u=0, \\
\partial \bar{y} & =\frac{1}{2}[x, x]-[x, u], \quad \partial \bar{v}=\frac{1}{2}[u, u]-[x, u], \\
\partial t & =[x, \bar{v}]-[u, \bar{y}] .
\end{aligned}
$$

Conjecture 3.3 on the model for $F(M, 2)$ is then confirmed by comparing to the model of Kř́ž ([]) for projective varieties.

\section{REFERENCES}

[1] Glen E. Bredon, Topology and geometry, Graduate Texts in Mathematics, vol. 139, SpringerVerlag, New York, 1997. Corrected third printing of the 1993 original. MR.1700700 (2000b:55001)

[2] O. Burlet, Rational homotopy of oriented Thom spaces, Proceedings of the Advanced Study Institute on Algebraic Topology (Aarhus Univ., Aarhus, 1970), Mat. Inst., Aarhus Univ., Aarhus, 1970, pp. 20-22. Various Publ. Ser., No. 13. MR0336743 (49 \#1516)

[3] Albrecht Dold, Lectures on algebraic topology, Classics in Mathematics, Springer-Verlag, Berlin, 1995. Reprint of the 1972 edition. MR.1335915 (96c:55001)

[4] Yves Félix, Stephen Halperin, and Jean-Claude Thomas, Rational homotopy theory, Graduate Texts in Mathematics, vol. 205, Springer-Verlag, New York, 2001. MR 1802847(2002d:55014) 
[5] Yves Félix, John Oprea, and Daniel Tanré, Algebraic models in geometry, Oxford Graduate Texts in Mathematics, vol. 17, Oxford University Press, Oxford, 2008. MR2403898 (2009a:55006)

[6] Igor Kříž, On the rational homotopy type of configuration spaces, Ann. of Math. (2) 139 (1994), no. 2, 227-237, DOI 10.2307/2946581. MR.1274092 (95c:55012)

[7] Pascal Lambrechts and Don Stanley, The rational homotopy type of configuration spaces of two points (English, with English and French summaries), Ann. Inst. Fourier (Grenoble) 54 (2004), no. 4, 1029-1052. MR2111020(2005i:55016)

[8] Howard J. Marcum and Duane Randall, The homotopy Thom class of a spherical fibration, Proc. Amer. Math. Soc. 80 (1980), no. 2, 353-358, DOI 10.2307/2042976. MR577773 (81k:55026)

[9] John W. Milnor and James D. Stasheff, Characteristic classes, Annals of Mathematics Studies, No. 76, Princeton University Press, Princeton, N. J.; University of Tokyo Press, Tokyo, 1974. MR0440554 (55 \#13428)

[10] Ştefan Papadima, The rational homotopy of Thom spaces and the smoothing of homology classes, Comment. Math. Helv. 60 (1985), no. 4, 601-614, DOI 10.1007/BF02567434. MR826873 (87e:57030)

[11] Daniel Quillen, Rational homotopy theory, Ann. of Math. (2) 90 (1969), 205-295. MR.0258031 (41 \#2678)

[12] Dennis Sullivan, Infinitesimal computations in topology, Inst. Hautes Études Sci. Publ. Math. 47 (1977), 269-331 (1978). MR0646078 (58 \#31119)

[13] Daniel Tanré, Homotopie rationnelle: modèles de Chen, Quillen, Sullivan (French), Lecture Notes in Mathematics, vol. 1025, Springer-Verlag, Berlin, 1983. MR764769 (86b:55010)

[14] René Thom, Quelques propriétés globales des variétés différentiables (French), Comment. Math. Helv. 28 (1954), 17-86. MR0061823 (15,890a)

Département de Mathématiques, Université Catholique de Louvain, 1348 Louvain-LANeuve, Belgique

E-mail address: yves.felix@uclouvain.be

Department of Mathematics, Cleveland State University, Cleveland, Ohio 44115

E-mail address: j.oprea@csuohio.edu

Département de Mathématiques, Université de Lille 1, 59655 Villeneuve D’AscQ Cedex, France

E-mail address: Daniel.Tanre@univ-lille1.fr 